\title{
The Dynamic Structure of Jadomycin B and the Amino Acid Incorporation Step of Its Biosynthesis
}

\author{
Uwe Rix, ${ }^{\natural}$ Jianting Zheng, ${ }^{\S}$ Lily L. Remsing Rix, \\ Rohr $^{\text {T* }}$ \\ Division of Pharmaceutical Sciences, College of Pharmacy, University of Kentucky, 907 Rose Street, Lexington, KY 40536-0082, \\ and Institute of Microbiology, Chinese Academy of Sciences, P.O. Box 2714, Beijing 100080, P.R. China. \\ Division of Pharmaceutical Sciences, College of Pharmacy, University of Kentucky, 907 Rose Street, Lexington, KY 40536-0082, \\ and Institute of Microbiology, Chinese Academy of Sciences, Beijing, P.R. China.
}

\section{Supporting Information}




\section{Cultivation of S. venezuelae ISP5230:}

A pre-culture of $100 \mathrm{~mL}$ liquid MYM (10 g/L malt extract, $4 \mathrm{~g} / \mathrm{L}$ yeast extract, 4 $\mathrm{g} / \mathrm{L}$ maltose; $\mathrm{pH}$ 7) medium in a $250 \mathrm{~mL}$ baffled Erlenmeyer shaker flask was inoculated with $40 \mu \mathrm{L}$ glycerol stock solution and grown for $24 \mathrm{~h}$ at $30^{\circ} \mathrm{C}$ and $200 \mathrm{rpm}$. Afterwards, $2 \mathrm{~L}$ of the main culture $(40 \times 50 \mathrm{~mL}$ in $250 \mathrm{~mL}$ baffled shaker flasks, I(2)-G medium, see below), was inoculated with $3 \mathrm{~mL}$ of the pre-culture per flask. After $13 \mathrm{~h}\left(30^{\circ} \mathrm{C}, 200\right.$ rpm) of growth each flask was supplemented with $2 \mathrm{~mL}$ of $100 \%$ ethanol and growth was continued for approximately $48 \mathrm{~h}$. The culture was then harvested and the mycelium was separated from the supernatant culture medium by vacuum filtration. The mycelium was subsequently re-suspended with acetone and the cells were lysed by sonification. The supernatant was separated from the cell debris by vacuum filtration and acetone was removed under reduced pressure. The remaining aqueous phase was recombined with the culture filtrate and extracted with ethyl acetate. The extracts were evaporated, re-dissolved in methanol, filtered and examined by analytical HPLC/MS, using the following solvent system: Solvent $A=0.1 \%$ formic acid in $\mathrm{H}_{2} \mathrm{O}$; solvent $\mathrm{B}=$ acetonitrile; flow rate $=0.5 \mathrm{ml} / \mathrm{min}$; $\min 0-6: 75 \% \mathrm{~A}$ and $25 \% \mathrm{~B}$ to $100 \% \mathrm{~B}$ (linear gradient); min 6-7: $100 \%$ B; min 7-7.5: $100 \%$ B to $75 \%$ A and $25 \%$ B (linear gradient); $\min 7.5-10: 75 \% \mathrm{~A}$ and $25 \% \mathrm{~B}$. The MS spectrometer was set to scan a mass/charge ratio from 200 to 750 , switching continuously between the positive and negative $\mathrm{APCl}$ mode. Purification was achieved by semipreparative HPLC: Solvent $A=0.1 \%$ trifluoroacetic acid in $\mathrm{H}_{2} \mathrm{O}$; solvent $\mathrm{B}=$ acetonitrile; flow rate $=10.0 \mathrm{~mL} / \mathrm{min}$; $\min 0-20$ : $50 \% A$ and $50 \%$ B to $100 \%$ B (linear gradient); $\min 20-23: 100 \%$ B; $\min 23-23.5: 100 \%$ B to $50 \% A$ and $50 \%$ B (linear gradient); $\min 23.5-29.5: 50 \% A$ and $50 \%$ B. Due to the 
acid sensitivity of jadomycin B (5), eluted fractions were immediately neutralized with $1 \mathrm{M}$ phosphate buffer ( $\mathrm{pH}$ 7.0). Subsequently, the organic solvent was evaporated and the remaining aqueous phase was extracted with ethyl acetate and concentrated in vacuum to dryness. The final purification step was performed via column chromatography on Sephadex LH-20 (Methanol).

\section{I(2)-G-medium}

$7.8 \mathrm{~g} \quad$ L-isoleucine $(59.5 \mathrm{mmol} / \mathrm{L})$

$1.16 \mathrm{~g} \quad \mathrm{KH}_{2} \mathrm{PO}_{4}$

$0.5 \mathrm{~g} \quad \mathrm{MgSO}_{4}$

$0.2 \mathrm{~g} \quad \mathrm{~K}_{2} \mathrm{HPO}_{4}$

$9 \mathrm{ml} \quad$ salt solution

$4.5 \mathrm{ml} \quad$ mineral solution

$4.5 \mathrm{ml} \quad \mathrm{FeSO}_{4} \times 7 \mathrm{H}_{2} \mathrm{O}(0.2 \% \mathrm{w} / \mathrm{v})$

De-ionized water was added to a final volume of $900 \mathrm{ml}$. After autoclavation, $100 \mathrm{ml}$ of sterile $30 \%(\mathrm{w} / \mathrm{v})$ aqueous galactose was added.

Salt solution:

$1 \%(\mathrm{w} / \mathrm{v}) \quad \mathrm{NaCl}$

$1 \%(\mathrm{w} / \mathrm{v}) \quad \mathrm{CaCl}_{2}$

Mineral Solution:

$880 \mathrm{mg} \quad \mathrm{ZnSO}_{4} \times 7 \mathrm{H}_{2} \mathrm{O}$

$39 \mathrm{mg} \mathrm{CuSO}_{4} \times 5 \mathrm{H}_{2} \mathrm{O}$

$6.1 \mathrm{mg} \quad \mathrm{MnSO}_{4} \times 4 \mathrm{H}_{2} \mathrm{O}$

$5.7 \mathrm{mg} \quad \mathrm{H}_{3} \mathrm{BO}_{3}$

$3.7 \mathrm{mg} \quad\left(\mathrm{NH}_{4}\right)_{6} \mathrm{Mo}_{7} \mathrm{O}_{24} \times 4 \mathrm{H}_{2} \mathrm{O}$

De-ionized water was added to a final volume of $1 \mathrm{~L}$.

Generation of jadomycin B (5) analogues: 
S. venezuelae ISP5230 was cultivated as described above with the exception that Lisoleucine in the main culture medium was completely replaced by the appropriate amount (59.5 $\mathrm{mmol} / \mathrm{L})$ of the according L-amino acid.

\section{Jadomycin B (5)}

Yield: $12 \mathrm{mg} / \mathrm{L} ; \mathrm{R}_{\text {rel }}=7.55 \mathrm{~min}$ (analytical)

$\mathrm{M}=549 \mathrm{~g} / \mathrm{mol}\left(\mathrm{C}_{30} \mathrm{H}_{31} \mathrm{NO}_{9}\right)$

Positive mode ESI-MS: $\mathrm{m} / \mathbf{z}=572$ (60; high resolution: calc. for $\mathrm{C}_{30} \mathrm{H}_{31} \mathrm{NO}_{9} \mathrm{Na}$ : 572.1891, found: 572.189) $[\mathrm{M}+\mathrm{Na}]^{+}, 550$ (33; high resolution: calc. for $\mathrm{C}_{30} \mathrm{H}_{32} \mathrm{NO}_{9}$ : 550.2072, found: 550.208) $[\mathrm{M}+\mathrm{H}]^{+}, 528(78)\left[\mathrm{M}+\mathrm{Na}-\mathrm{CO}_{2}\right]^{+}, 506(5)\left[\mathrm{M}+\mathrm{H}-\mathrm{CO}_{2}\right]^{+}, 458$ (30) $\left[\mathrm{M}+\mathrm{Na}-\mathrm{C}_{6} \mathrm{H}_{10} \mathrm{O}_{2}\right]^{+}, 420$ (65) $[\mathrm{M}+\mathrm{H} \text {-digitoxose }]^{+}, 306$ (100) $[\mathrm{M}+\mathrm{H}$-digitoxose$\left.\mathrm{C}_{6} \mathrm{H}_{10} \mathrm{O}_{2}\right]^{+}$.

IR (KBr): v = 3493 (br, m), 2950 (sh, m), 2926 (s, s), 2854, 1806 (sh, m), 1669 (sh, m), 1577, 1516 (sh, s), 1458 (sh, s), 1376 (sh, m), 1272 (sh, s), 1235 (sh, m), 1103, 1058, 974 (sh, m) cm ${ }^{-1}$.

UV/Vis (Methanol): $\lambda_{\max }(\epsilon)=533$ (900), 399 (1200), 313 (7600), 289 (sh), 235 (7000), $212(13500) \mathrm{nm}$. 
${ }^{1} \mathrm{H}-\mathrm{NMR}$ (400 MHz, $\mathrm{d}_{6}$-acetone, TMS), and ${ }^{13} \mathrm{C}-\mathrm{NMR}$ (100 MHz, $\mathrm{d}_{6}$-acetone, TMS) of Jadomycin B (5).

Form I (1S, 3aS, 1'S) 67\%

\begin{tabular}{|c|c|c|c|c|c|}
\hline Position & $\delta^{1} \mathrm{H}$ [ppm] & $\begin{array}{c}\text { Multiplicity } \\
(\mathrm{J}[\mathrm{Hz}])\end{array}$ & $\begin{array}{c}\delta^{13} \mathrm{C} \\
{[\mathrm{ppm}]}\end{array}$ & HMBC & NOESY \\
\hline 1 & 5.41 & $d(3)$ & 63.2 & $2,3 a, 1^{\prime}, 2^{\prime}, 4^{\prime}$ & $2 ' a, 3 a, 3^{\prime}$ \\
\hline 2 & & & 171.0 & & \\
\hline $3 a$ & 6.31 & $\mathrm{~S}$ & 88.1 & $2,4,13 a$ & 1,4 \\
\hline $3 b$ & & & $119.7^{e}$ & & \\
\hline 4 & 6.77 & $\mathrm{~s}(\mathrm{br})$ & 114.6 & $3 a, 6,5-\mathrm{CH}_{3}$ & $5-\mathrm{CH}_{3}$ \\
\hline 5 & & & 143.0 & & \\
\hline $5-\mathrm{CH}_{3}$ & 2.36 & $\mathrm{~S}$ & 21.2 & $4,5,6$ & 4,6 \\
\hline 6 & 6.83 & s (br) & 120.9 & $4,5-\mathrm{CH}_{3}, 7,7 \mathrm{a}$ & $5-\mathrm{CH}_{3}$ \\
\hline 7 & & & 155.0 & & \\
\hline $7-\mathrm{OH}$ & 10.30 & $\mathrm{~s}(\mathrm{br})$ & & 6,7 & \\
\hline $7 a$ & & & 112.2 & & \\
\hline $7 b$ & & & $138.7^{e}$ & & \\
\hline 8 & & & 184.1 & & \\
\hline $8 a$ & & & 136.4 & & \\
\hline 9 & 7.91 & $\mathrm{dd}(7.5,1.5)$ & 121.8 & $8,10,12 a$ & \\
\hline 10 & 7.86 & $\mathrm{dd}(8.5,7.5)$ & 137.2 & $8 a, 9,11,12$ & \\
\hline 11 & 7.67 & $\mathrm{dd}(8.5,1.5)$ & 121.4 & $9,10,12,12 a, 13$ & 1" \\
\hline 12 & & & 157.1 & & \\
\hline $12 \mathrm{a}$ & & & 118.7 & & \\
\hline 13 & & & 181.1 & & \\
\hline $13 a$ & & & 131.5 & & \\
\hline 1 ' & 1.97 & M & 40.0 & $1,2,2^{\prime}$ & \\
\hline 2'a & 1.86 & M & 26.2 & $1,1^{\prime}, 3^{\prime}, 4^{\prime}$ & 1 \\
\hline$\beta$ & 1.53 & M & & $1,1^{\prime}, 3^{\prime}, 4^{\prime}$ & \\
\hline 3 & 1.08 & $t(7.5)$ & 12.3 & $1^{\prime}, 2^{\prime}$ & 1 \\
\hline $4 '$ & 0.48 & $\mathrm{~d}(7)$ & 14.5 & $1,1^{\prime}, 2^{\prime}$ & \\
\hline $1 "$ & 6.09 & d (3) & 95.9 & $12,3 "$ & 11 \\
\hline $2 "{ }_{a x}$ & 2.28 & ddd $(15,3.5,3)$ & 36.1 & $1 "$ & \\
\hline eq & 2.41 & Ddd $(15,3,1)$ & & 1",3",4" & \\
\hline 3"' & 4.02 & $M$ & 66.9 & 1",2",4",5" & \\
\hline 4" & 3.21 & $\operatorname{Dd}(10,3.5)$ & 73.3 & 5", $5 "-\mathrm{CH}_{3}$ & \\
\hline 5" & 3.76 & $\mathrm{dq}(10,6)$ & 66.7 & 4",5"- $-\mathrm{CH}_{3}$ & \\
\hline 5"- $-\mathrm{CH}_{3}$ & 1.17 & $d(6)$ & 18.3 & 4",5" & \\
\hline
\end{tabular}


Form II (1S, 3aR, 1'S) 33\%

\begin{tabular}{|c|c|c|c|c|c|}
\hline Position & $\delta^{1} \mathrm{H}$ [ppm] & $\begin{array}{c}\text { Multiplicity } \\
(\mathrm{J}[\mathrm{Hz}])\end{array}$ & $\begin{array}{c}\delta{ }^{13} \mathrm{C} \\
{[\mathrm{ppm}]}\end{array}$ & HMBC & NOESY \\
\hline 1 & $\overline{5.41}$ & $d(3)$ & 63.3 & $2,3 a, 1^{\prime}, 2^{\prime}, 4^{\prime}$ & 2 'a \\
\hline 2 & & & 170.3 & & \\
\hline $3 a$ & 6.72 & $\mathrm{~s}$ & 88.5 & 4,7 & 4',2"' eq \\
\hline $3 b$ & & & $121.2^{\mathrm{e}}$ & & \\
\hline 4 & 6.74 & $\mathrm{~s}(\mathrm{br})$ & 115.8 & $5-\mathrm{CH}_{3}, 6,7 \mathrm{a}$ & \\
\hline 5 & & & 142.2 & & \\
\hline $5-\mathrm{CH}_{3}$ & 2.33 & $s$ & 21.1 & $4,5,6$ & \\
\hline 6 & 6.76 & $\mathrm{~s}(\mathrm{br})$ & 121.0 & $4,7,7 a$ & \\
\hline 7 & & & 154.9 & & \\
\hline $7-\mathrm{OH}$ & 10.93 & $s$ & & & \\
\hline $7 a$ & & & 111.9 & & \\
\hline $7 b$ & & & $145.2^{\mathrm{e}}$ & & \\
\hline 8 & & & 182.4 & & \\
\hline $8 a$ & & & 136.4 & & \\
\hline 9 & 7.80 & $m^{*}$ & 121.3 & 8,10 & \\
\hline 10 & 7.79 & $\mathrm{~m}^{*}$ & 137.3 & $8 a, 12$ & \\
\hline 11 & 7.58 & $\mathrm{~m}^{*}$ & 120.6 & 9,12 & $1 "$ \\
\hline 12 & & & 156.2 & & \\
\hline $12 a$ & & & 119.4 & & \\
\hline 13 & & & 183.1 & & \\
\hline $13 a$ & & & 128.6 & & \\
\hline 1 ' & 2.19 & $\mathrm{~m}$ & 39.4 & & \\
\hline 2'a & 1.86 & $\mathrm{~m}$ & 27.2 & 1,1',3',4' & \\
\hline$\beta$ & 1.53 & $\mathrm{~m}$ & & $1,1^{\prime}, 3^{\prime}, 4^{\prime}$ & $4 '$ \\
\hline 3 & 1.11 & $t(7.5)$ & 12.2 & $1^{\prime}, 2^{\prime}$ & \\
\hline 4' & 1.07 & $\mathrm{~d}(7)$ & 15.0 & $1,1^{\prime}, 2^{\prime}$ & $3 a, 2$ ' $\beta$ \\
\hline 1" & 5.99 & d (3) & 96.4 & $12,3 "$ & 11 \\
\hline $2 " a x$ & 2.28 & ddd $(15,3.5,3)$ & 36.3 & $1 "$ & \\
\hline eq & 2.37 & ddd $(15,3,1)$ & & 1",3",4" & $3 a$ \\
\hline 3" & 4.02 & $\mathrm{~m}$ & 67.3 & 1",2",4",5" & \\
\hline $4 "$ & 3.21 & dd $(10,3.5)$ & 73.4 & 5", 5"'-CH & \\
\hline $5 "$ & 3.87 & $\mathrm{dq}(10,6)$ & 66.7 & 4",5"- $-\mathrm{CH}_{3}$ & \\
\hline 5"- $-\mathrm{CH}_{3}$ & 1.21 & $d(6)$ & 18.3 & 4",5" & \\
\hline
\end{tabular}

exchangeable, *higher order effect 


\section{Jadomycin V (6)}

Yield: $5 \mathrm{mg} / \mathrm{L} ; \mathrm{R}_{\mathrm{rel}}=7.18 \mathrm{~min}$ (analytical)

$\mathrm{M}=535 \mathrm{~g} / \mathrm{mol}\left(\mathrm{C}_{29} \mathrm{H}_{29} \mathrm{NO}_{9}\right)$

Positive mode APCI-MS: $\mathrm{m} / \mathrm{z}=536(53)[\mathrm{M}+\mathrm{H}]^{+}, 492(100)\left[\mathrm{M}+\mathrm{H}-\mathrm{CO}_{2}\right]^{+}, 406(85)[\mathrm{M}+\mathrm{H}-$ digitoxose $]^{+}, 306(55)\left[\mathrm{M}+\mathrm{H}-\mathrm{C}_{6} \mathrm{H}_{10} \mathrm{O}_{2}\right]^{+}$.

UV/Vis (Acetonitrile/ $\mathrm{H}_{2} \mathrm{O}$ ): $\lambda_{\max }=397,313,292(\mathrm{sh}), 240,212 \mathrm{~nm}$.

${ }^{1} \mathrm{H}-\mathrm{NMR}\left(400 \mathrm{MHz}, \mathrm{d}_{6}\right.$-acetone, TMS) of Jadomycin V (6).

Form I (1S, 3aS) $66 \%$

Form II $(1 S, 3 a R) 34 \%$

\begin{tabular}{|c|c|c|c|c|c|c|}
\hline $\begin{array}{c}\text { Positio } \\
n\end{array}$ & $\begin{array}{c}\delta^{1} \mathbf{H} \\
{[\mathrm{ppm}} \\
]\end{array}$ & $\begin{array}{l}\text { Multiplicity } \\
(\mathrm{J}[\mathrm{Hz}])\end{array}$ & COSY & $\begin{array}{c}\delta^{1} H \\
{[\mathrm{ppm}} \\
]\end{array}$ & $\begin{array}{c}\text { Multiplicity } \\
(\mathrm{J}[\mathrm{Hz}])\end{array}$ & COSY \\
\hline 1 & 5.28 & $\mathrm{~d}(3)$ & $1^{\prime}$ & 5.37 & $\mathrm{~d}(3.5)$ & $1^{\prime}$ \\
\hline $3 a$ & 6.30 & $\mathrm{~s}(\mathrm{br})$ & & 6.77 & $\mathrm{~s}$ & \\
\hline 4 & 6.78 & $M$ & $5-\mathrm{CH}_{3}, 6$ & 6.78 & $\mathrm{~m}$ & $5-\mathrm{CH} 3,6$ \\
\hline $5-\mathrm{CH}_{3}$ & 2.37 & $\mathrm{~S}$ & 4,6 & 2.35 & $\mathrm{~s}$ & 4 \\
\hline 6 & 6.84 & M & $5-\mathrm{CH}_{3}, 4$ & 6.81 & $\mathrm{~m}$ & 4 \\
\hline $7-\mathrm{OH}$ & 10.33 & $\mathrm{~s}(\mathrm{br})^{\mathrm{D}}$ & & 10.95 & $\mathrm{~s}(\mathrm{br})^{\mathrm{D}}$ & \\
\hline 9 & 7.94 & $\mathrm{dd}(7.5,1.5)$ & 10,11 & 7.85 & $\mathrm{~m}^{*}$ & 10,11 \\
\hline 10 & 7.87 & $\mathrm{dd}(8.5,7.5)$ & 9,11 & 7.84 & $\mathrm{~m}^{*}$ & 9,11 \\
\hline 11 & 7.67 & $\mathrm{dd}(8.5,1.5)$ & 9,10 & 7.62 & $\mathrm{dd}(7.5,2.5)$ & 9,10 \\
\hline $1^{\prime}$ & 2.21 & M & $1,2^{\prime}, 3^{\prime}$ & 2.48 & $\mathrm{~m}$ & 1,2',3' \\
\hline 2' & 0.54 & d (7) & 1 & 1.13 & $\mathrm{~d}(7)$ & $1 '$ \\
\hline $3{ }^{\prime}$ & 1.25 & $d(7)$ & $1^{\prime}$ & 1.31 & $d(7)$ & $1^{\prime}$ \\
\hline $1 "$ & 6.13 & d (3) & 2" & 6.03 & d (3) & $2{ }^{\prime \prime}$ ax \\
\hline $2 "$ "ax & 2.28 & ddd $(15,3.5,3)$ & 1",2" eq, 3" & 2.28 & ddd $(15,3.5,3)$ & 1",2" ${ }_{\text {eq }}, 3$ " \\
\hline eq & 2.42 & ddd $(15,3,1.5)$ & 2" ax & 2.37 & ddd $(15,3,1.5)$ & $2{ }^{\prime \prime a x}$ \\
\hline 3" & 4.02 & $\mathrm{M}$ & $2{ }^{\prime \prime}, 4 "$ & 4.02 & $\mathrm{~m}$ & 2"'ax,4" \\
\hline 4" & 3.22 & $\mathrm{dd}(10,3.5)$ & 3",5" & 3.22 & $\mathrm{dd}(10,3.5)$ & 3",5" \\
\hline $5 "$ & 3.75 & $\mathrm{dq}(10,6.5)$ & 4",5"--CH & 3.87 & $\mathrm{dq}(10,6.5)$ & 4", $5 "-\mathrm{CH}_{3}$ \\
\hline 5"- $-\mathrm{CH}_{3}$ & 1.17 & $d(6)$ & $5 "$ & 1.19 & $d(6)$ & 5" \\
\hline
\end{tabular}

Dexchangeable with $\mathrm{D}_{2} \mathrm{O}$, *higher order effect 


\section{Jadomycin F (7)}

Yield: $10 \mathrm{mg} / \mathrm{L} ; \mathrm{R}_{\mathrm{rel}}=7.13 \mathrm{~min}$ (analytical)

$\mathrm{M}=583 \mathrm{~g} / \mathrm{mol}\left(\mathrm{C}_{33} \mathrm{H}_{29} \mathrm{NO}_{9}\right)$

Positive mode APCI-MS: $\mathrm{m} / \mathrm{z}=584$ (36; ESI high resolution: calc. for $\mathrm{C}_{33} \mathrm{H}_{30} \mathrm{NO}_{9}$ : 584.1915, found: 584.1917) $[\mathrm{M}+\mathrm{H}]^{+}, 540(100)\left[\mathrm{M}+\mathrm{H}-\mathrm{CO}_{2}\right]^{+}, 454(18)[\mathrm{M}+\mathrm{H} \text {-digitoxose }]^{+}$, $410(22)\left[\mathrm{M}+\mathrm{H} \text {-digitoxose- } \mathrm{CO}_{2}\right]^{+}, 306(35)\left[\mathrm{M}+\mathrm{H} \text {-digitoxose- } \mathrm{C}_{9} \mathrm{H}_{8} \mathrm{O}_{2}\right]^{+}$.

IR (KBr): v = 3468 (br, m), 2928 (br, w), 1804, 1684 (sh, s), 1777, 1516 (sh, w), 1454 (sh, m), 1381 (sh, w), 1273 (sh, w), 1211 (sh, s), 1136 (sh, s), 970 (sh, m), 843 (sh, m), 804 (sh, m), 726 (sh, m) cm ${ }^{-1}$.

UV/Vis (Methanol): $\lambda_{\max }(\epsilon)=497$ (1000), 393 (1400), 310 (9300), 289 (sh), 239 (9100), $211(13000) \mathrm{nm}$.

\footnotetext{
${ }^{1} \mathrm{H}-\mathrm{NMR}\left(400 \mathrm{MHz}, \mathrm{d}_{6}\right.$-acetone, TMS) and ${ }^{13} \mathrm{C}-\mathrm{NMR}\left(100 \mathrm{MHz}, \mathrm{d}_{6}\right.$-acetone, TMS) of Jadomycin $\mathrm{F}(\mathbf{7})$.
}

Form I (1S, 3aS) $60 \%$

\begin{tabular}{|c|c|c|c|c|c|}
\hline Position & $\delta^{1} \mathbf{H}[\mathbf{p p m}]$ & $\begin{array}{c}\text { Multiplicity } \\
(\mathbf{J}[\mathbf{H z}])\end{array}$ & $\begin{array}{c}\delta^{13} \mathbf{C} \\
{[\mathbf{p p m}]}\end{array}$ & HMBC & COSY \\
\hline 1 & 5.65 & $\mathrm{dd}(5,3)$ & 61.8 & & $1^{\prime} \mathrm{a}, 1^{\prime} \beta$ \\
\hline 2 & & & 171.9 & & \\
\hline $3 \mathrm{a}$ & 6.08 & $\mathrm{~S}$ & 88.0 & $3 \mathrm{~b}, 7 \mathrm{a}$ & \\
\hline $3 \mathrm{~b}$ & & & $119.6^{\mathrm{e}}$ & & \\
\hline 4 & 6.06 & $\mathrm{~s}(\mathrm{br})$ & 113.8 & $\begin{array}{c}3 \mathrm{a}, 3 \mathrm{~b}, 5- \\
\mathrm{CH}_{3}, 7 \mathrm{a}\end{array}$ & 6 \\
\hline 5 & & & 142.9 & & \\
\hline $5-\mathrm{CH}_{3}$ & 2.22 & $\mathrm{~S}$ & 21.1 & $4,5,6$ & \\
\hline 6 & 6.69 & $\mathrm{~S}(\mathrm{br})$ & 119.9 & $4,5-\mathrm{CH}_{3}, 7,7 \mathrm{a}$ & 4 \\
\hline 7 & & & 154.9 & & \\
\hline
\end{tabular}




\begin{tabular}{|c|c|c|c|c|c|}
\hline $7-\mathrm{OH}$ & 10.19 & $\mathrm{~s}(\mathrm{br})$ & & & \\
\hline $7 a$ & & & 112.1 & & \\
\hline $7 b$ & & & $135.4^{\mathrm{e}}$ & & \\
\hline 8 & & & 184.2 & & \\
\hline $8 a$ & & & 135.9 & & \\
\hline 9 & 7.95 & $\mathrm{dd}(7.5,1)$ & 121.4 & $8,12 a$ & 10 \\
\hline 10 & 7.89 & $\mathrm{dd}(8.5,7.5)$ & 137.3 & $8 a, 9,11,12$ & 9,11 \\
\hline 11 & 7.70 & $\mathrm{dd}(8.5,1)$ & 120.9 & $9,12 a$ & 10 \\
\hline 12 & & & 157.2 & & \\
\hline $12 a$ & & & 118.6 & & \\
\hline 13 & & & 181.7 & & \\
\hline $13 a$ & & & 131.5 & & \\
\hline 1'a & $3.26-3.36$ & $M^{*}$ & 39.2 & $3^{\prime}, 7^{\prime}$ & 1 \\
\hline$\beta$ & $3.26-3.36$ & $M^{*}$ & & $3^{\prime}, 7^{\prime}$ & 1 \\
\hline 2 & & & 136.6 & & \\
\hline $3^{\prime}$ & 7.36 & $M^{*}$ & 130.8 & $5^{\prime}, 7^{\prime}$ & 4' \\
\hline $4 '$ & $7.21-7.31$ & $M^{*}$ & 129.8 & $6^{\prime}$ & $3^{\prime}, 5^{\prime}$ \\
\hline 5 & 6.90 & $\mathrm{M}^{*}$ & 127.9 & & 4',6',7' \\
\hline $6^{\prime}$ & $7.21-7.31$ & $M^{*}$ & 129.8 & 4' & 5 \\
\hline $7^{\prime}$ & 6.79 & $M^{*}$ & 130.6 & & 5 \\
\hline $1 "$ & 6.16 & d (3.5) & 95.8 & $3 "$ & 2" \\
\hline 2" $a x$ & 2.29 & ddd $(15.5,3.5,3)$ & 36.0 & $3 "$ & 1",2" eq \\
\hline eq & 2.44 & ddd $(15.5,3.5,1)$ & & 3",4" & $2 "{ }_{a x}$ \\
\hline 3"' & 4.15 & $\mathrm{~m}$ & 67.0 & $1 "$ & 4" \\
\hline 4" & 3.23 & $\mathrm{dd}(10,3.5)$ & 73.3 & 5"- $-\mathrm{CH}_{3}$ & 3",5" \\
\hline 5" & 3.77 & $\mathrm{dq}(10,6)$ & 66.7 & 5"- $-\mathrm{CH}_{3}$ & 4", $5 "-\mathrm{CH}_{3}$ \\
\hline 5"- $-\mathrm{CH}_{3}$ & 1.17 & $d(6)$ & 18.3 & 4",5" & $5 "$ \\
\hline
\end{tabular}

Form II (1S, 3aR) 40\%

\begin{tabular}{|c|c|c|c|c|c|}
\hline Position & $\begin{array}{c}\delta^{\mathbf{1}} \mathbf{H} \\
{[\mathbf{p p m}]}\end{array}$ & $\begin{array}{c}\text { Multiplicity } \\
(\mathbf{J}[\mathbf{H z}])\end{array}$ & $\begin{array}{c}\delta^{13} \mathbf{C} \\
{[\mathbf{p p m}]}\end{array}$ & HMBC & COSY \\
\hline 1 & 5.68 & $\mathrm{dd}(7,3.5)$ & 61.0 & $3 \mathrm{a}, 2^{\prime}$ & $1^{\prime} \mathrm{a}, 1^{\prime} \beta$ \\
\hline 2 & & & 171.0 & & \\
\hline $3 \mathrm{a}$ & 5.93 & $\mathrm{~s}$ & 87.9 & & \\
\hline $3 \mathrm{~b}$ & & & $121.3^{\mathrm{e}}$ & & \\
\hline 4 & 6.67 & $\mathrm{~s}(\mathrm{br})$ & 116.1 & $5-\mathrm{CH}_{3}, 7 \mathrm{a}$ & 6 \\
\hline 5 & & & 142.5 & & \\
\hline $5-\mathrm{CH} \mathrm{H}_{3}$ & 2.31 & $\mathrm{~s}$ & 21.1 & $4,5,6$ & \\
\hline 6 & 6.80 & $\mathrm{~s}(\mathrm{br})$ & 121.5 & $4,5-\mathrm{CH}_{3}, 7 \mathrm{a}$ & 4 \\
\hline 7 & & & 155.2 & & \\
\hline $7-\mathrm{OH}$ & 10.86 & $\mathrm{~s}$ & & & \\
\hline $7 \mathrm{a}$ & & & 112.0 & & \\
\hline
\end{tabular}




\begin{tabular}{|c|c|c|c|c|c|}
\hline $7 b$ & & & $136.3^{\mathrm{e} 1}$ & & \\
\hline 8 & & & 182.5 & & \\
\hline $8 a$ & & & 136.3 & & \\
\hline 9 & 7.88 & $M^{*}$ & 121.9 & $8,8 \mathrm{a}$ & 10 \\
\hline 10 & 7.87 & $M^{*}$ & 137.5 & $8 a, 9,11,12$ & 9,11 \\
\hline 11 & 7.67 & $M^{*}$ & 121.2 & 9,10 & 10 \\
\hline 12 & & & 156.5 & & \\
\hline $12 a$ & & & 120.0 & & \\
\hline 13 & & & 182.8 & & \\
\hline $13 a$ & & & 128.6 & & \\
\hline 1 'a & 3.41 & $M^{*}$ & 36.6 & & 1 \\
\hline$\beta$ & 3.47 & $M^{*}$ & & $3^{\prime}, 7^{\prime}$ & 1 \\
\hline $2^{\prime}$ & & & 134.8 & & \\
\hline $3{ }^{\prime}$ & 7.36 & $M^{*}$ & 130.8 & $5^{\prime}, 7^{\prime}$ & $?$ \\
\hline 4' & 7.21-7.31 & $M^{*}$ & $129.6^{\mathrm{e} 2}$ & $6^{\prime}$ & $?$ \\
\hline 5 & 6.83 & $M^{*}$ & 128.5 & $2^{\prime}$ & $?$ \\
\hline $6^{\prime}$ & $7.21-7.31$ & $\mathrm{M}^{*}$ & $129.7^{\mathrm{e} 2}$ & $4 '$ & $?$ \\
\hline $7^{\prime}$ & 6.82 & $M^{*}$ & 128.6 & $2^{\prime}$ & $?$ \\
\hline 1" & 6.07 & d (3.5) & 96.4 & 2",3" & 2"'ax \\
\hline $2 "$ ax & 2.31 & ddd $(15.5,3.5,3)$ & 36.2 & & $1 ", 2$ "eq \\
\hline eq & 2.47 & ddd $(15.5,3.5,1.5)$ & & 3",4" & $2 "$ ax \\
\hline 3"' & 4.15 & $\mathrm{~m}$ & 67.3 & & 4" \\
\hline 4" & 3.28 & dd $(10,3.5)$ & 73.4 & 5"- $-\mathrm{CH}_{3}$ & $3 ", 5 "$ \\
\hline 5" & 3.88 & $d q(10,6)$ & 66.7 & 5"- $-\mathrm{CH}_{3}$ & 4",5"--CH 3 \\
\hline 5"- $-\mathrm{CH}_{3}$ & 1.20 & $d(6)$ & 18.4 & 4",5" & 5" \\
\hline
\end{tabular}

exchangeable, *higher order effect

\section{Jadomycin ALA (8)}

Yield: $6 \mathrm{mg} / \mathrm{L} ; \mathrm{R}_{\mathrm{rel}}=5.87 \mathrm{~min}$ (analytical)

$\mathrm{M}=507 \mathrm{~g} / \mathrm{mol}\left(\mathrm{C}_{27} \mathrm{H}_{25} \mathrm{NO}_{9}\right)$

Positive mode APCI-MS: $\mathrm{m} / \mathrm{z}=508$ (48; ESI high resolution: calc. for $\mathrm{C}_{27} \mathrm{H}_{25} \mathrm{NO}_{9}$ : 508.1602, found: 508.1612) $[\mathrm{M}+\mathrm{H}]^{+}, 464(100)\left[\mathrm{M}+\mathrm{H}-\mathrm{CO}_{2}\right]^{+}, 436(7)\left[\mathrm{M}+\mathrm{H}-\mathrm{CO}_{2}-\mathrm{CO}\right]^{+}$, 378 (12) $[\mathrm{M}+\mathrm{H} \text {-digitoxose }]^{+}, 334(13)\left[\mathrm{M}+\mathrm{H} \text {-digitoxose- } \mathrm{CO}_{2}\right]^{+}, 306(47)[\mathrm{M}+\mathrm{H}$-digitoxose$\left.\mathrm{C}_{3} \mathrm{H}_{4} \mathrm{O}_{2}\right]^{+}$. 
UV/Vis (Methanol): $\lambda_{\max }(\varepsilon)=495$ (500), 399 (600), 311 (3400), 290 (sh), 240 (3500), $213(8700) \mathrm{nm}$.

${ }^{1} \mathrm{H}$-NMR (400 MHz, $\mathrm{d}_{6}$-acetone, TMS) and ${ }^{13} \mathrm{C}$-NMR (100 MHz, $\mathrm{d}_{6}$-acetone, TMS) of Jadomycin ALA (8).

Form I (1S, 3aS) 47\%

\begin{tabular}{|c|c|c|c|c|}
\hline Position & $\delta{ }^{1} \mathrm{H}$ [ppm] & $\begin{array}{l}\text { Multiplicity } \\
(\mathrm{J}[\mathrm{Hz}])\end{array}$ & $\begin{array}{c}\delta{ }^{13} \mathrm{C} \\
{[\mathrm{ppm}]}\end{array}$ & COSY \\
\hline 1 & 5.32 & $q(6.5)$ & 55.7 & $1-\mathrm{CH}_{3}$ \\
\hline $1-\mathrm{CH}_{3}$ & 1.55 & $d(6.5)$ & 19.8 & 1 \\
\hline 2 & & & 172.4 & \\
\hline $3 a$ & 6.39 & $\mathrm{~s}(\mathrm{br})$ & 88.3 & \\
\hline $3 b$ & & & 119.2 & \\
\hline 4 & 6.82 & $\mathrm{~s}(\mathrm{br})^{\circ}$ & 114.4 & \\
\hline 5 & & & 142.9 & \\
\hline $5-\mathrm{CH}_{3}$ & 2.37 & $\mathrm{~s}$ & 20.7 & \\
\hline 6 & 6.84 & $\mathrm{~s}(\mathrm{br})^{0}$ & 120.9 & \\
\hline 7 & & & 154.9 & \\
\hline $7-\mathrm{OH}$ & 10.29 & $\mathrm{~s}(\mathrm{br})$ & & \\
\hline $7 a$ & & & 115.8 & \\
\hline $7 b$ & & & N.O. & \\
\hline 8 & & & 184.3 & \\
\hline $8 a$ & & & 136.0 & \\
\hline 9 & 7.94 & dd $(8,1)$ & 121.5 & 10,11 \\
\hline 10 & 7.87 & $\mathrm{dd}(8.5,8)$ & 136.8 & 9,11 \\
\hline 11 & 7.68 & $\mathrm{dd}(8.5,1)$ & 121.3 & 9,10 \\
\hline 12 & & & 156.8 & \\
\hline $12 a$ & & & 118.5 & \\
\hline 13 & & & N.O. & \\
\hline $13 a$ & & & N.O. & \\
\hline 1 ' & 6.11 & $d(3)$ & 96.0 & $2_{a x}^{\prime}$ \\
\hline $2_{a x}^{\prime}$ & 2.29 & ddd $(15,3.5,3)$ & 36.0 & 1',2'eq,3' \\
\hline eq & 2.43 & $\operatorname{ddd}(15,3.5,1)$ & & $2_{a x}^{\prime}, 3^{\prime}, 4^{\prime}$ \\
\hline $3^{\prime}$ & 4.01 & $\mathrm{~m}$ & 66.9 & $2^{\prime}$ ax, $2^{\prime}$ eq, $3^{\prime}$ \\
\hline $4 '$ & 3.22 & $\mathrm{dd}(10,3)$ & 73.2 & 3,5 \\
\hline 5 & 3.76 & $d q(10,6)$ & 66.5 & $4^{\prime}, 5^{\prime}-\mathrm{CH}_{3}$ \\
\hline $5^{\prime}-\mathrm{CH}_{3}$ & 1.14 & $\mathrm{D}(6)$ & 17.9 & $5{ }^{\prime}$ \\
\hline
\end{tabular}


Form II (1S, 3aR) 53\%

\begin{tabular}{|c|c|c|c|c|}
\hline Position & $\delta^{1} \mathrm{H}[\mathrm{ppm}]$ & $\begin{array}{l}\text { Multiplicity } \\
(\mathrm{J}[\mathrm{Hz}])\end{array}$ & $\begin{array}{c}\delta^{13} \mathrm{C} \\
{[\mathrm{ppm}]}\end{array}$ & COSY \\
\hline 1 & 5.42 & Q (7) & 55.9 & $1-\mathrm{CH}_{3}$ \\
\hline $1-\mathrm{CH}_{3}$ & 1.67 & $\mathrm{D}(7)$ & 15.1 & 1 \\
\hline 2 & & & 172.0 & \\
\hline $3 a$ & 6.80 & $\mathrm{~s}(\mathrm{br})^{\mathrm{O}}$ & 87.1 & \\
\hline $3 b$ & & & 117.0 & \\
\hline 4 & 6.80 & $\mathrm{~s}(\mathrm{br})^{\circ}$ & 116.4 & \\
\hline 5 & & & 141.7 & \\
\hline $5-\mathrm{CH}_{3}$ & 2.35 & $\mathrm{~s}$ & 20.7 & \\
\hline 6 & 6.82 & $\mathrm{~s}(\mathrm{br})^{\mathrm{O}}$ & 121.3 & \\
\hline 7 & & & 159.0 & \\
\hline $7-\mathrm{OH}$ & 10.95 & S (br) & & \\
\hline $7 a$ & & & 112.0 & \\
\hline $7 b$ & & & N.O. & \\
\hline 8 & & & 180.2 & \\
\hline $8 a$ & & & 136.1 & \\
\hline 9 & 7.87 & $\mathrm{dd}(7.5,1.5)$ & 121.2 & 10,11 \\
\hline 10 & 7.86 & $\mathrm{dd}(8,7.5)$ & 137.1 & 9,11 \\
\hline 11 & 7.59 & $\mathrm{dd}(8,1.5)$ & 120.9 & 9,10 \\
\hline 12 & & & 155.8 & \\
\hline $12 a$ & & & 119.1 & \\
\hline 13 & & & N.O. & \\
\hline $13 a$ & & & N.O. & \\
\hline 1 & 6.05 & $d(3)$ & 95.8 & \\
\hline $2_{\text {ax }}^{\prime}$ & 2.27 & ddd $(15,3.5,3)$ & 36.0 & 1',2', ', 3' \\
\hline eq & 2.39 & ddd $(15,3.5,1)$ & & $2_{a x}^{\prime}, 3^{\prime}, 4^{\prime}$ \\
\hline 3 & 4.01 & $\mathrm{~m}$ & 66.9 & $2_{a x}^{\prime}, 2_{\text {eq }}^{\prime}, 3^{\prime}$ \\
\hline $4 '$ & 3.22 & dd $(10,3)$ & 73.2 & 3,5 \\
\hline 5 & 3.85 & $d q(10,6)$ & 66.4 & $4^{\prime}, 5^{\prime}-\mathrm{CH}_{3}$ \\
\hline $5^{\prime}-\mathrm{CH}_{3}$ & 1.18 & $d(6)$ & 17.9 & $5^{\prime}$ \\
\hline
\end{tabular}

N.O. 'not observed', 'overlapping signals

Jadomycin T (9),

3aR-diastereomer (> 95\%)

Yield: $9.5 \mathrm{mg} / \mathrm{L} ; \mathrm{R}_{\text {rel }}=6.92 \mathrm{~min}$ (analytical) 
$\mathrm{M}=537 \mathrm{~g} / \mathrm{mol}\left(\mathrm{C}_{28} \mathrm{H}_{27} \mathrm{NO}_{10}\right)$

Positive mode APCI-MS: $\mathrm{m} / \mathrm{z}=538$ (100; ESI high resolution: calc. for $\mathrm{C}_{28} \mathrm{H}_{28} \mathrm{NO}_{10}$ : 538.1708, found: 538.1709) $[\mathrm{M}+\mathrm{H}]^{+}, 494(11)\left[\mathrm{M}+\mathrm{H}-\mathrm{CO}_{2}\right]^{+}, 408(37)[\mathrm{M}+\mathrm{H} \text {-digitoxose }]^{+}$, 306 (35) $\left[\mathrm{M}+\mathrm{H} \text {-digitoxose }-\mathrm{C}_{4} \mathrm{H}_{6} \mathrm{O}_{3}\right]^{+}$.

IR (KBr): v = 3473 (br, m), 2926 (sh, m), 2745, 1690 (sh, s), 1578 (sh, w), 1517 (sh, m), 1458 (s, m), 1385 (sh, w), 1275 (sh, m), 1207 (sh, s), 1134 (sh, m), 976 (sh, m) cm cm. $^{-1}$

UV/Vis (Methanol): $\lambda_{\max }(\epsilon)=501$ (300), 408 (700), 317 (3800), 292 (3700), 240 (4200), $211(5700) \mathrm{nm}$.

${ }^{1} \mathrm{H}-\mathrm{NMR}\left(400 \mathrm{MHz}, \mathrm{d}_{6}\right.$-acetone, TMS) and ${ }^{13} \mathrm{C}-\mathrm{NMR}$ (100 MHz, $\mathrm{d}_{6}$-acetone, TMS) of Jadomycin T (9).

$\left(1 S, 3 a R, 1^{\prime} R\right)$

\begin{tabular}{|c|c|c|c|c|}
\hline Position & $\delta{ }^{1} \mathbf{H}$ [ppm] & $\begin{array}{c}\text { Multiplicity } \\
(\mathbf{J}[\mathrm{Hz}])\end{array}$ & $\delta^{{ }^{13} \mathbf{C}}$ [ppm] & CYCLENOE \\
\hline 1 & 4.68 & $\mathrm{dd}(8,1)$ & 69.4 & $1^{\prime}-\mathrm{CH}_{3}$ \\
\hline 2 & & & 173.3 & \\
\hline $3 \mathrm{a}$ & 5.87 & $\mathrm{~s}(\mathrm{br})$ & 89.4 & $4,1^{\prime}$ \\
\hline $3 \mathrm{~b}$ & & & $120.6^{\mathrm{e}}$ & \\
\hline 4 & 6.77 & $\mathrm{~s}(\mathrm{br})$ & 116.1 & $3 \mathrm{a}$ \\
\hline 5 & & & 141.3 & \\
\hline $5-\mathrm{CH}_{3}$ & 2.31 & $\mathrm{~S}$ & 21.1 & \\
\hline 6 & 6.76 & $\mathrm{~s}(\mathrm{br})$ & 120.5 & \\
\hline 7 & & & 154.9 & \\
\hline $7-\mathrm{OH}$ & 11.02 & $\mathrm{~s} \mathrm{(br)})^{\mathrm{D}}$ & & \\
\hline $7 \mathrm{a}$ & & & 112.8 & \\
\hline $7 \mathrm{~b}$ & & & $149.1^{\mathrm{e}}$ & \\
\hline 8 & & & 182.8 & \\
\hline $8 \mathrm{a}$ & & & $137.1^{\mathrm{e}}$ & \\
\hline 9 & 7.90 & $\mathrm{dd}(8.5,1)$ & 121.4 & \\
\hline
\end{tabular}




\begin{tabular}{|c|c|c|c|c|}
\hline 10 & 7.83 & dd $(9,8.5)$ & 137.2 & \\
\hline 11 & 7.54 & dd $(9,1)$ & 120.9 & \\
\hline 12 & & & 156.4 & \\
\hline $12 a$ & & & 118.3 & \\
\hline 13 & & & 181.0 & \\
\hline $13 a$ & & & 130.8 & \\
\hline $1^{\prime}$ & 4.37 & $\mathrm{dq}(8,7)$ & 79.8 & $3 a$ \\
\hline 1'- $-\mathrm{CH}_{3}$ & 1.55 & d (7) & 20.1 & 1 \\
\hline 1'-OH & 3.30 & $\mathrm{~s}^{\mathrm{D}}$ & & \\
\hline 1" & 6.02 & d (3) & 95.8 & \\
\hline $2 "$ ax & 2.26 & ddd $(15,3,3)$ & 35.6 & \\
\hline eq & 2.40 & $\operatorname{ddd}(15,3,1)$ & & \\
\hline 3" & 4.15 & $M$ & 67.6 & \\
\hline $4 "$ & 3.38 & $\mathrm{dd}(10,3.5)$ & 73.4 & \\
\hline $5 "$ & 3.89 & $\mathrm{dq}(10,6.5)$ & 65.8 & \\
\hline 5"- $-\mathrm{CH}_{3}$ & 1.17 & $d(6.5)$ & 18.4 & \\
\hline
\end{tabular}

\section{Jadomycin T - 3aS-diastereomer (<5\%)}

Yield : $0.4 \mathrm{mg} / \mathrm{L} ; \mathrm{R}_{\mathrm{rel}}=6.08 \mathrm{~min}$ (analytical)

$\mathrm{M}=537 \mathrm{~g} / \mathrm{mol}\left(\mathrm{C}_{28} \mathrm{H}_{27} \mathrm{NO}_{10}\right)$

ESI high resolution: calc. for $\mathrm{C}_{28} \mathrm{H}_{27} \mathrm{NO}_{10} \mathrm{Na:} 560.1527$, found: 560.1532 .

Positive mode APCI-MS: m/z = $538(39)[\mathrm{M}+\mathrm{H}]^{+}, 408$ (63) $[\mathrm{M}+\mathrm{H} \text {-digitoxose }]^{+}, 366(100)$ $\left[\mathrm{M}+\mathrm{H}-\mathrm{C}_{2} \mathrm{H}_{2} \mathrm{O}\right]^{+}, 306(35)\left[\mathrm{M}+\mathrm{H} \text {-digitoxose- } \mathrm{C}_{4} \mathrm{H}_{6} \mathrm{O}_{3}\right]^{+}$.

UV/Vis (Acetonitrile/ $\left.\mathrm{H}_{2} \mathrm{O}\right): \lambda_{\max }(\epsilon)=401,314,292(\mathrm{sh}), 240(\mathrm{sh}), 212 \mathrm{~nm}$. 
Jadomycin S (10),

3aR-diastereomer (>95\%)

Yield: $7 \mathrm{mg} / \mathrm{L} ; \mathrm{R}_{\mathrm{rel}}=7.19 \mathrm{~min}$ (analytical)

$\mathrm{M}=523 \mathrm{~g} / \mathrm{mol}\left(\mathrm{C}_{27} \mathrm{H}_{25} \mathrm{NO}_{10}\right)$

Positive mode APCI-MS: $\mathrm{m} / \mathrm{z}=524$ (100; ESI high resolution: calc. for $\mathrm{C}_{27} \mathrm{H}_{26} \mathrm{NO}_{10}$ : 524.1551, found: 524.1536) $[\mathrm{M}+\mathrm{H}]^{+}, 480(44)\left[\mathrm{M}+\mathrm{H}-\mathrm{CO}_{2}\right]^{+}, 436(4)\left[\mathrm{M}+\mathrm{H}-\mathrm{C}_{4} \mathrm{H}_{6} \mathrm{O}_{3}\right], 394$ (80) $[\mathrm{M}+\mathrm{H} \text {-digitoxose }]^{+}, 306(62)\left[\mathrm{M}+\mathrm{H} \text {-digitoxose }-\mathrm{C}_{4} \mathrm{H}_{6} \mathrm{O}_{3}\right]^{+}$.

IR (KBr): v = 3462 (br, s), 2927 (sh, m), 1685 (br, s), 1577, 1519 (sh, m), 1457 (sh, m), 1393, 1275 (sh, m), 1206 (sh, s), 1136 (sh, s), 972 (sh, m), 843 (sh, m), 804 (sh, m), 725 (sh, m) $\mathrm{cm}^{-1}$.

UV/Vis (Methanol): $\lambda_{\max }(\varepsilon)=560$ (600), 503 (400), 397 (700), 316 (4500), 292 (4300), 240 (4200), $212(6400) \mathrm{nm}$.

${ }^{1} \mathrm{H}-\mathrm{NMR}\left(400 \mathrm{MHz}, \mathrm{d}_{6}\right.$-acetone, TMS) and ${ }^{13} \mathrm{C}-\mathrm{NMR}$ (100 MHz, $\mathrm{d}_{6}$-acetone, TMS) of Jadomycin $S$ (10)

$(1 S, 3 a R) 100 \%$

\begin{tabular}{|c|c|c|c|}
\hline Position & $\delta{ }^{\mathbf{1}} \mathbf{H}[\mathbf{p p m}]$ & $\begin{array}{c}\text { Multiplicity } \\
(\mathbf{J}[\mathrm{Hz}])\end{array}$ & $\delta^{{ }^{13}} \mathbf{C}$ [ppm] \\
\hline 1 & 5.30 & ddd $(7.5,5,1)$ & 62.7 \\
\hline 2 & & & 173.6 \\
\hline $3 a$ & 5.84 & $\mathrm{~s}(\mathrm{br})$ & 89.8 \\
\hline $3 \mathrm{~b}$ & & & $122.9^{\mathrm{e}}$ \\
\hline 4 & 6.79 & $\mathrm{~s}(\mathrm{br})$ & 115.3 \\
\hline 5 & & & 141.7 \\
\hline $5-\mathrm{CH}_{3}$ & 2.33 & $\mathrm{~S}$ & 21.2 \\
\hline 6 & 6.76 & $\mathrm{~s}(\mathrm{br})$ & 120.3 \\
\hline 7 & & & 154.8 \\
\hline
\end{tabular}




\begin{tabular}{|c|c|c|c|}
\hline $7-\mathrm{OH}$ & 10.94 & $\mathrm{~s}(\mathrm{br})$ & \\
\hline $7 a$ & & & 112.8 \\
\hline $7 b$ & & & 148.2 \\
\hline 8 & & & 182.5 \\
\hline $8 a$ & & & $137.0^{\mathrm{e}}$ \\
\hline 9 & 7.90 & $\mathrm{dd}(7.5,1)$ & 121.5 \\
\hline 10 & 7.82 & $\mathrm{dd}(8.5,7.5)$ & 137.2 \\
\hline 11 & 7.56 & $\mathrm{dd}(8.5,1)$ & 120.4 \\
\hline 12 & & & 156.4 \\
\hline $12 \mathrm{a}$ & & & 118.4 \\
\hline 13 & & & 181.9 \\
\hline $13 a$ & & & 131.4 \\
\hline $1^{\prime} \alpha$ & 4.32 & $\mathrm{dd}(8.5,5)$ & 71.2 \\
\hline$\beta$ & 4.55 & $\mathrm{dd}(8.5,7.5)$ & \\
\hline $1 "$ & 6.07 & $d(3)$ & 95.4 \\
\hline 2" "ax & 2.26 & ddd $(15,3.5,3.5)$ & 35.6 \\
\hline eq & 2.40 & ddd $(15,3,1)$ & \\
\hline $3 "$ & 4.14 & ddd $(3.5,3.5,3)$ & 67.4 \\
\hline 4" & 3.36 & $\mathrm{dd}(9.5,3)$ & 73.3 \\
\hline $5 "$ & 3.86 & $\mathrm{dq}(9.5,6.5)$ & 65.9 \\
\hline 5"- $-\mathrm{CH}_{3}$ & 1.16 & $d(6.5)$ & 18.3 \\
\hline
\end{tabular}

Jadomycin S - 3aS-diastereomer $(<5 \%)$

Yield : $0.3 \mathrm{mg} / \mathrm{L} ; \mathrm{R}_{\mathrm{rel}}=5.66 \mathrm{~min}$ (analytical)

$\mathrm{M}=523 \mathrm{~g} / \mathrm{mol}\left(\mathrm{C}_{27} \mathrm{H}_{25} \mathrm{NO}_{10}\right)$

Positive mode APCI-MS: $\mathrm{m} / \mathrm{z}=524(61)[\mathrm{M}+\mathrm{H}]^{+}, 480(5)\left[\mathrm{M}+\mathrm{H}-\mathrm{CO}_{2}\right]^{+}, 394(100)[\mathrm{M}+\mathrm{H}-$ digitoxose $]^{+}, 306(45)\left[\mathrm{M}+\mathrm{H} \text {-digitoxose- } \mathrm{C}_{4} \mathrm{H}_{6} \mathrm{O}_{3}\right]^{+}$.

UV/Vis (Methanol): $\lambda_{\max }=395,316,292,240,212 \mathrm{~nm}$. 
HPLC/MS analyses of jadomycin B (5) analogues generated by feeding of various L-amino acids to the S. venezuelae ISP5230 wild-type.

\begin{tabular}{|c|c|c|c|c|c|c|}
\hline $\begin{array}{l}\text { Added Amino } \\
\text { Acid }^{\mathrm{a}}\end{array}$ & $\mathbf{R}_{\text {rel }}$ & $\mathbf{X}$ & $\underset{\text { Mass }^{b}}{\Delta}$ & \multicolumn{3}{|c|}{$\begin{array}{l}\text { Jadomycin X } \\
\mathrm{MS}[\mathrm{m} / \mathrm{z}]^{\mathrm{c}}\end{array}$} \\
\hline Isoleucine & 7.55 & B & - & 549 & $550,506,420,306$ & $399,313,289,235,212$ \\
\hline $\begin{array}{l}\text { Alanine } \\
\text { Valine } \\
\text { Leucine }\end{array}$ & $\begin{array}{l}5.87 \\
7.18 \\
7.35\end{array}$ & $\begin{array}{l}\text { ALA } \\
\text { V } \\
\text { L }\end{array}$ & $\begin{array}{l}-42 \\
-14 \\
+/-0\end{array}$ & $\begin{array}{l}507 \\
535 \\
549\end{array}$ & $\begin{array}{l}508,464,378,306 \\
536,492,406,306 \\
550,506,420,306\end{array}$ & $\begin{array}{l}399,311,290,240,213 \\
402,314,289,244 \\
398,312,292,240,213\end{array}$ \\
\hline $\begin{array}{l}\text { Phenylalanine } \\
\text { Tyrosine } \\
\text { Tryptophan } \\
\end{array}$ & $\begin{array}{l}7.13 \\
5.66 \\
6.17 \\
\end{array}$ & $\begin{array}{l}F \\
Y \\
W\end{array}$ & $\begin{array}{l}+34 \\
+50 \\
+73 \\
\end{array}$ & $\begin{array}{l}583 \\
599 \\
622 \\
\end{array}$ & $\begin{array}{l}584,540,454,306 \\
600,556,470,306 \\
623,493,447 \\
\end{array}$ & $\begin{array}{l}393,310,289,239,211 \\
407,314,292,229,213 \\
397,318,295,244,210\end{array}$ \\
\hline $\begin{array}{l}\text { Serine (3a- } R \text { ) } \\
\text { Threonine (3a- } R \text { ) }\end{array}$ & $\begin{array}{l}7.19 \\
6.92\end{array}$ & $\begin{array}{l}\mathbf{S} \\
T\end{array}$ & $\begin{array}{l}-26 \\
-12\end{array}$ & $\begin{array}{l}523 \\
537\end{array}$ & 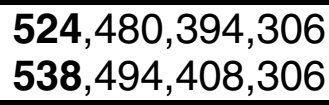 & $\begin{array}{l}396,311,292,247 \\
408,317,292,240,211\end{array}$ \\
\hline Methionine & 6.58 & M & +18 & 567 & $568,524,438,306$ & $397,310,289,241,218$ \\
\hline $\begin{array}{l}\text { Asparagine } \\
\text { Histidine }\end{array}$ & $\begin{array}{l}4.75 \\
3.44\end{array}$ & $\begin{array}{l}\mathbf{N} \\
\mathrm{H}\end{array}$ & $\begin{array}{r}+1 \\
+24 \\
\end{array}$ & $\begin{array}{l}550 \\
573\end{array}$ & 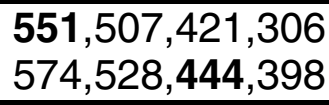 & $\begin{array}{l}394,313,293,239,215 \\
392,310,236,211\end{array}$ \\
\hline
\end{tabular}

${ }^{a}$ all amino acids used were of L-configuration ${ }^{b}$ expected mass difference in comparison to jadomycin $\mathrm{B}{ }^{\mathrm{C}} \mathrm{MS}$ data are positive mode APCI, all values correspond to the protonated ion species; MS base peaks (100\%) are depicted in bold; blue highlighted compounds were only investigated with HPLC/MS

MS data shown represent the major fragmentation patterns: -44 (loss of $\mathrm{CO}_{2}$ ); -130 loss of (L-digitoxose); ' 306 ' represents phenanthroviridone obtained by loss of the amino acid side chain and of L-digitoxose

\section{Molecular Modeling:}

The Maestro 5.1.016 software was used, with the Merck forcefield. 